\title{
SYNTHESIS OF QUINOXALINE 1,4-DI- $N$-OXIDE DERIVATIVES ON SOLID SUPPORT USING ROOM TEMPERATURE AND MICROWAVE-ASSISTED SOLVENT-FREE PROCEDURES
}

Lilia C. Gómez-Caro, Mario Sánchez-Sánchez, Virgilio Bocanegra-García and Gildardo Rivera*

Departamento de Farmacia y Quimica Medicinal, Universidad Autónoma de Tamaulipas, 88740, Reynosa, México

Antonio Monge

Unidad de Investigación y Desarrollo de Medicamentos, Centro de Investigación en Farmacobiologia Aplicada, Universidad de Navarra, 31008, Pamplona, España

Recebido em 19/8/10; aceito em 16/2/11; publicado na web em 15/4/11

\begin{abstract}
We describe the synthesis of 12 new ethyl and methyl quinoxaline-7-carboxylate 1,4-di- $N$-oxide derivatives on solid supports with room temperature and microwave-assisted solvent-free procedures. Results show that solid supports have good catalytic activity in the formation of quinoxaline 1,4-di- $N$-oxide derivatives. We found that florisil and montmorillonite KSF and K10 could be used as new, easily available, inexpensive alternatives of catalysts. Additionally, room temperature and microwave-irradiation solvent-free synthesis was more efficient than a conventional procedure (Beirut reaction), reducing reaction time and increasing yield.
\end{abstract}

Keywords: quinoxaline; solid supports; solvent-free.

\section{INTRODUCTION}

Quinoxaline has numerous biological properties and has been studied as an anti-leishmanial, anti-fungal, anti-bacterial, anti-Chagas, and anti-tumoral agent. ${ }^{1-4}$ Quinoxaline 1,4-di- $N$-oxide is a versatile scaffold with diverse activities (Figure 1). ${ }^{5-8}$<smiles>N#Cc1c(N)[n+]([O-])c2ccccc2[n+]1[O-]</smiles>

anti-tumoral agent<smiles>[R]c1ccc(C2CC(c3c(C)[n+]([O-])c4ccc([R])cc4[n+]3[O-])=NN2)cc1</smiles>

Figure 1. Structure of some active quinoxaline 1,4-di-N-oxide derivatives

A conventional procedure known as the Beirut reaction has been used for the synthesis of quinoxaline 1,4-di- $N$-oxide by various authors. The reaction mechanism involves the presence of triethylamine as a base capable of removing a proton from $\alpha$-methylene transferring it to the carbonyl group of the 2,4-diketone, forming a carbanion, which performs a nucleophilic attack on the positive nitrogen of benzofuroxane. Subsequently, there is a nucleophilic attack of a second nitrogen on the carbonyl group with the resulting cyclization and reorganization binding (Figure 2); however, this procedure has several limitations such as a low yield and a prolonged reaction

*e-mail: gildardors@ hotmail.com time. ${ }^{9-12}$ Therefore, the development of new catalysts for the synthesis of quinoxaline 1,4-di- $N$-oxide derivatives is highly desirable.<smiles>[R]C(=O)CC([R])=O</smiles><smiles></smiles>

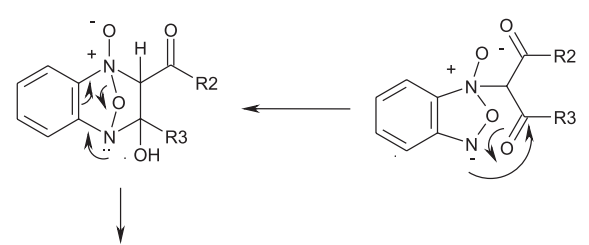<smiles>[R2]C(=O)c1c([R3])[n+]([O-])c2ccccc2[n+]1[O-]</smiles>

Figure 2. Synthesis and reaction mechanism of quinoxaline 1,4-di-N-oxide by the Beirut reaction

The use of solid supports in various chemical syntheses has recently been documented. ${ }^{13-15}$ Additionally, some solid supports with catalytic activity, such as montmorillonite KSF and K10, have been widely studied as reusable catalysts. ${ }^{16-18}$ Several methods have 
been reported for the synthesis of quinoxaline derivatives using solid supports and microwave-assisted reactions. ${ }^{19-24}$

The aim of this study was the synthesis of new ethyl and methyl quinoxaline-7-carboxylate 1,4-di- $N$-oxide derivatives on solid supports such as silica gel, florisil, alumina, $4 \AA$ molecular sieves, montmorillonite KSF, and montmorillonite K10, by room temperature and microwave-assisted solvent-free procedures (Figure 3), as a strategy to enhance yield and reduce the reaction time as a part of our ongoing research in the design and synthesis of new compounds with potential pharmacological activity.

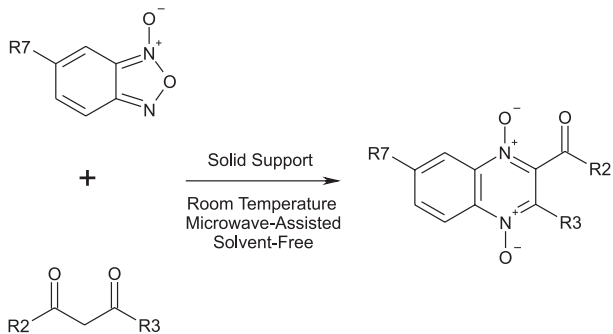

Figure 3. General procedure for the synthesis of new ethyl and methyl quinoxaline-7-carboxylate 1,4-di-N-oxide derivatives on solid supports by room temperature and microwave-assisted solvent-free procedures

\section{RESULTS AND DISCUSSION}

As a part of our ongoing research in the synthesis of new antiChagas, anti-tumoral, and anti-mycobacterial agents, we developed 12 new ethyl and methyl quinoxaline-7-carboxylate 1,4-di- $N$-oxide derivatives using a previously reported conventional procedure (the Beirut reaction $)^{11,12}$ shown in Table 1 . Unfortunately, we obtained ethyl and methyl quinoxaline-7-carboxylate 1,4-di- $N$-oxide derivatives with a low yield (0.4-23.2\%). Some authors have used the Beirut reaction to synthesize various heterocyclic compounds by reacting benzofuroxane with enolates and enamines reporting good to excellent yields; however, when $\beta$-keto esters and diketones in basic medium (triethylamine) are used, low yields of quinoxaline 1,4-di- $N$ oxide derivatives are obtained. ${ }^{9}{ }^{10}$ Considering the generation of the carbanion on $\alpha$-methylene as a key step in the reaction mechanism that forms quinoxaline, we propose that there was greater difficulty in generating the carbanion on $\beta$-keto ester derivatives by effect resonance, which may explain the low yields in entry 4 and 5 and the
Table 1. Ethyl and methyl quinoxaline-7-carboxylate 1,4-di- $N$-oxide derivatives obtained by conventional procedure (Beirut reaction)<smiles>[R]C(=O)c1c([R3])[n+](O)c2ccc([R])cc2[n+]1[O-]</smiles>

\begin{tabular}{ccccc}
\hline Entry & $\mathrm{R} 2$ & $\mathrm{R} 3$ & $\mathrm{R} 7$ & Yield \% \\
\hline 1 & $\mathrm{C}_{6} \mathrm{H}_{5}$ & $\mathrm{CH}_{3}$ & $\mathrm{CH}_{3} \mathrm{OOC}$ & 10 \\
2 & $\mathrm{NHC}_{6} \mathrm{H}_{5}$ & $\mathrm{CH}_{3}$ & $\mathrm{CH}_{3} \mathrm{OOC}$ & 5.04 \\
3 & $\mathrm{OCH}_{3}$ & $\mathrm{CH}_{3}$ & $\mathrm{CH}_{3} \mathrm{OOC}$ & 0 \\
4 & $\mathrm{OCH}_{3} \mathrm{CH}_{2}$ & $\mathrm{CH}_{3}$ & $\mathrm{CH}_{3} \mathrm{OOC}$ & 1.50 \\
5 & $\left.\mathrm{OC} \mathrm{CH}_{3}\right)_{3}$ & $\mathrm{CH}_{3}$ & $\mathrm{CH}_{3} \mathrm{OOC}$ & 0.4 \\
6 & $\mathrm{C}_{6} \mathrm{H}_{5}$ & $\mathrm{CF}_{3}$ & $\mathrm{CH}_{3} \mathrm{OOC}$ & 10.9 \\
7 & $\mathrm{C}_{4} \mathrm{H}_{3} \mathrm{~S}$ & $\mathrm{CF}_{3}$ & $\mathrm{CH}_{3} \mathrm{OOC}$ & 5.6 \\
8 & $\mathrm{C}_{6} \mathrm{H}_{5}$ & $\mathrm{CH}_{3}$ & $\mathrm{CH}_{3} \mathrm{CH}_{2} \mathrm{OOC}$ & 12.3 \\
9 & $\mathrm{NHC}_{6} \mathrm{H}_{5}$ & $\mathrm{CH}_{3}$ & $\mathrm{CH}_{3} \mathrm{CH}_{2} \mathrm{OOC}$ & 22.4 \\
10 & $\mathrm{OCH}_{3}$ & $\mathrm{CH}_{3}$ & $\mathrm{CH}_{3} \mathrm{CH}_{2} \mathrm{OOC}$ & 0 \\
11 & $\mathrm{C}_{6} \mathrm{H}_{5}$ & $\mathrm{CF}_{3}$ & $\mathrm{CH}_{3} \mathrm{CH}_{2} \mathrm{OOC}$ & 2.3 \\
12 & $\mathrm{C}_{4} \mathrm{H}_{3} \mathrm{~S}$ & $\mathrm{CF}_{3}$ & $\mathrm{CH}_{3} \mathrm{CH}_{2} \mathrm{OOC}$ & 23.2 \\
\hline
\end{tabular}

lack of compounds in 3 and 10. This behavior could also explain the low yield in aromatic diketones, but this conclusion is not definite and needs further experimentation.

As an alternative strategy in the synthesis of quinoxaline 1,4-di- $N$-oxide derivatives, we proposed the use of solid supports such as silica gel, alumina and $4 \AA$ molecular sieves that have been reported to have catalytic activity and enhance yield in the synthesis of quinoxaline 1,4 -di- $N$-oxide. ${ }^{25}$ We included florisil, montmorillonite KSF and K10 as new solid supports with potential catalytic activity. The reaction system starting with methyl benzofuroxane-5-carboxylate and 1-benzoylacetone (entry 1) without the addition of solid supports and monitored with thin layer chromatography at different times for $48 \mathrm{~h}$, did not proceed, but when solid supports from a room temperature solvent-free procedure were used, we obtained compounds like those synthesized by the conventional procedure with an enhanced yield and a shorter reaction time (Table 2).

Table 2. Ethyl and methyl quinoxaline-7-carboxylate 1,4-di- $N$-oxide derivatives obtained by room temperature and microwave-assisted solvent-free procedures

\begin{tabular}{|c|c|c|c|c|c|c|c|c|c|c|c|c|}
\hline \multirow{2}{*}{ Entry } & \multicolumn{6}{|c|}{ Room temperature $(\%)$} & \multicolumn{6}{|c|}{ Microwave-assisted (\%) } \\
\hline & $\mathrm{F}$ & $\mathrm{A}$ & $\mathrm{S}$ & MS & $\mathrm{KSF}$ & K10 & $\mathrm{F}$ & $\mathrm{A}$ & $\mathrm{S}$ & MS & KSF & K10 \\
\hline 1 & $70 *$ & $40^{*}$ & $25^{*}$ & $20 \approx$ & $25^{*}$ & $25^{\approx}$ & $60^{\mathrm{a}}$ & $50^{\mathrm{a}}$ & $70^{\mathrm{c}}$ & $50^{\mathrm{b}}$ & $65^{b}$ & $55^{b}$ \\
\hline 2 & $40 *$ & $20^{\approx}$ & $20^{=}$ & $0^{*}$ & $20^{\approx}$ & $25^{\approx}$ & $60^{\mathrm{a}}$ & $50^{\mathrm{b}}$ & $40^{\mathrm{b}}$ & $20^{\mathrm{c}}$ & $50^{\mathrm{b}}$ & $30^{\mathrm{a}}$ \\
\hline 3 & $60 *$ & $30^{\approx}$ & $0 *$ & $0^{*}$ & $0 *$ & $0^{*}$ & $40^{\mathrm{a}}$ & $60^{c}$ & $0^{\mathrm{c}}$ & $20^{\mathrm{c}}$ & $0^{\mathrm{c}}$ & $0^{\mathrm{c}}$ \\
\hline 4 & $40^{\approx}$ & $15^{*}$ & $0^{*}$ & $80^{\approx}$ & $0^{*}$ & $0 *$ & $80^{\mathrm{a}}$ & $70^{c}$ & $0^{c}$ & $80^{\mathrm{c}}$ & $35^{\mathrm{b}}$ & $10^{\mathrm{c}}$ \\
\hline 5 & $20 *$ & $20 *$ & $0^{*}$ & $0^{*}$ & $0^{*}$ & $30 *$ & $40^{\mathrm{b}}$ & $50^{\mathrm{a}}$ & $40^{\mathrm{b}}$ & $25^{b}$ & $20^{\mathrm{b}}$ & $0^{\mathrm{c}}$ \\
\hline 6 & $30 *$ & $20^{\approx}$ & $20 *$ & $5^{\approx}$ & $0^{*}$ & $5^{\approx}$ & $30^{\mathrm{a}}$ & $30^{\mathrm{b}}$ & $35^{\mathrm{a}}$ & $20^{\mathrm{a}}$ & $15^{\mathrm{c}}$ & $20^{\mathrm{a}}$ \\
\hline 7 & $20^{=}$ & $20^{\approx}$ & $40 *$ & $20^{\approx}$ & $20^{\approx}$ & $20^{\approx}$ & $20^{\mathrm{c}}$ & $30^{\mathrm{a}}$ & $40^{c}$ & $25^{\mathrm{a}}$ & $35^{\mathrm{c}}$ & $55^{\mathrm{b}}$ \\
\hline 8 & $40^{\approx}$ & $40^{=}$ & $35^{-}$ & $10^{*}$ & $70^{\approx}$ & $90^{\approx}$ & $60^{\mathrm{a}}$ & $60^{\mathrm{a}}$ & $50^{\mathrm{c}}$ & $0^{\mathrm{c}}$ & $30^{b}$ & $20^{b}$ \\
\hline 9 & $60=$ & $30^{\approx}$ & $40^{=}$ & $40^{=}$ & $30^{\approx}$ & $50^{=}$ & $60^{\mathrm{a}}$ & $50^{\mathrm{a}}$ & $30^{\mathrm{a}}$ & $15^{\mathrm{a}}$ & $30^{\mathrm{b}}$ & $35^{\mathrm{a}}$ \\
\hline 10 & $50^{\approx}$ & $30^{*}$ & $20^{\approx}$ & $30^{=}$ & $30^{\approx}$ & $40^{\approx}$ & $30^{\mathrm{a}}$ & $20^{\mathrm{a}}$ & $40^{\mathrm{a}}$ & $15^{\mathrm{a}}$ & 25 & $40^{\mathrm{a}}$ \\
\hline 11 & $40^{\approx}$ & $60^{=}$ & $60^{=}$ & $60^{\approx}$ & $50^{\approx}$ & $60=$ & $70^{\mathrm{b}}$ & $60^{\mathrm{a}}$ & $80^{a}$ & $50^{\mathrm{a}}$ & 700 & $70^{\mathrm{a}}$ \\
\hline 12 & $50 \approx$ & $60^{\approx}$ & $20=$ & $5^{\approx}$ & $40^{\approx}$ & $20^{\approx}$ & $70^{\mathrm{c}}$ & $70^{\mathrm{a}}$ & $40^{\mathrm{a}}$ & $0^{\mathrm{c}}$ & $70^{\mathrm{b}}$ & $35^{\mathrm{c}}$ \\
\hline
\end{tabular}

$\mathrm{F}=$ Florisil, $\mathrm{A}=$ Alumina, $\mathrm{S}=$ Silica gel, $\mathrm{MS}=4 \AA$ Molecular sieves, $\mathrm{KSF}=$ Montmorillonite KSF, K10 = Montmorillonite K10. Time: ${ }^{1} 1 \mathrm{~h},{ }^{*} \geq 24 \mathrm{~h},{ }^{\text {a }} 5 \mathrm{~min}$,

${ }^{\mathrm{b}} 10 \mathrm{~min},{ }^{\mathrm{c}} 15 \mathrm{~min}$ and ${ }^{\mathrm{d}} 45 \mathrm{~min}$ 
Analyzing the data of entry 1 using a room temperature solventfree procedure, we found that there was a sevenfold and fourfold increase in yield with florisil $(70 \%)$ and alumina (40\%) as solid supports, respectively. This contrasted with the yield obtained by conventional procedure (10\%). When silica gel, $4 \AA$ molecular sieves, montmorillonite KSF and K10 were used, we obtained only a twofold yield (20\%). Although the results did not show a consistent pattern of enhanced yield with the other 2,4-diketone derivatives, this procedure was more efficient and effective than the conventional procedure, with florisil and alumina solid supports having the best catalytic activity. The yield increase obtained with solid supports may be due to the incorporation of 2,4-diketone derivatives on the surface of the solid supports, which allow better nucleophilic attack by the nitrogen of benzofuroxane as shown in Figure $4 .^{26}$ We propose that the nature of each solid support promotes the incorporation of 2,4-diketone derivatives more efficiently on the surface.
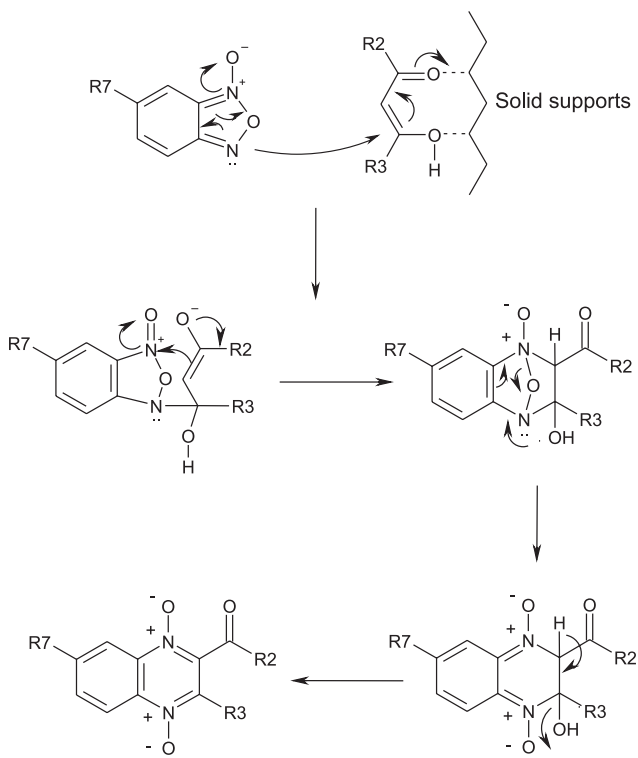

Figure 4. Reaction mechanism of quinoxaline 1,4-di-N-oxide on solid supports at room temperature

We unexpectedly found that in the reaction in entry 3,4 , and 5 using $\beta$-keto esters, the desired product was not obtained with silica gel, montmorillonite KSF or K10. This may be due to a greater interaction of the $\beta$-keto ester derivatives on solid supports, which prevents the formation of the quinoxaline derivative. ${ }^{27,28}$ This behavior may also explain the low yield obtained with florisil and alumina.

In a third strategy to obtain quinoxaline 1,4-di- $N$-oxide derivatives more efficiently, we carried out a microwave-assisted procedure using the same solid supports under solvent-free conditions (Table 2). In this procedure we obtained ethyl and methyl quinoxaline-7-carboxylate 1,4-di- $N$-oxide derivatives with a more efficient reaction time, 5 to 45 min versus $1 \mathrm{~h}$ and days with room temperature solvent-free and the conventional procedure, respectively. This short reaction time may be caused by the heating effect of microwaves. Although this heating effect is important, it should be considered that it can also cause the formation of undesired subproducts such as quinoxaline reduction derivatives. The catalytic activity in the condensation of benzofuroxane and $\beta$-keto esters and 2,4-diketone derivatives using the microwave-assisted procedure was more efficient, with florisil and alumina showing the best yields. Other solid supports also showed good yields, but our results with silica gel were different from those reported by Yusuke et al.. This was because they synthesized quinoxaline 1,4-di- $N$-oxide derivatives on silica gel in solvent-free conditions under microwave irradiation in only two minutes while we showed results in five minutes or more. ${ }^{29}$

Finally, our results show that florisil is a new solid support with catalytic activity and that montmorillonite KSF and K10 are additional alternatives for obtaining quinoxaline 1,4-di- $N$-oxide derivatives from benzofuroxane and $\beta$-ketoesters and 2,4-diketone derivatives.

\section{CONCLUSION}

In summary, our results show that solid supports have good catalytic activity in the formation of ethyl and methyl quinoxaline7-carboxylate 1,4-di- $N$-oxide derivatives. We found that florisil and montmorillonite KSF and K10 can be used as new, efficient, easily available, and inexpensive alternative catalysts. Additionally, the synthesis of quinoxaline 1,4-di- $\mathrm{N}$-oxide derivatives using room temperature and microwave-assisted solvent-free procedures is simpler, quick, and more efficient than a conventional procedure, reducing reaction time and increasing yield.

\section{EXPERIMENTAL}

\section{General procedure for the synthesis of quinoxaline 1,4-di- $N$ - oxide derivatives}

\section{Conventional procedure (Beirut reaction)}

We added $30 \mathrm{~mL}$ of dry chloroform, benzofuroxane derivative (5 mmol) and 2,4-diketone derivative $(10 \mathrm{mmol})$ at $0{ }^{\circ} \mathrm{C}$ to $50 \mathrm{~mL}$ round-bottomed flasks, and a catalytic amount of triethylamine and stirred the mixture at room temperature for 4-7 days. Progress of the reaction was monitored by thin layer chromatography. Afterwards, the obtained compound was purified by column chromatography using dichloromethane:methanol (95:5).

\section{Room temperature solvent-free procedure}

We added $20 \mathrm{~mL}$ of dry chloroform, benzofuroxane derivative (5 mmol), 2,4-diketone derivative $(10 \mathrm{mmol})$, and $500 \mathrm{mg}$ of solid support at room temperature to a $50 \mathrm{~mL}$ round-bottomed flask. After the reagents had dissolved, the solvent was then evaporated under reduced pressure and dried in a vacuum pump. Progress of the reaction was monitored by thin layer chromatography from 5 min to $24 \mathrm{~h}$. After completion of the reaction, we added dichloromethane, and the solid support was removed by filtration. The crude compound was purified by column chromatography using dichloromethane:methanol (95:5).

\section{Solvent-free using microwave-assisted procedure}

We added $20 \mathrm{~mL}$ of dry chloroform, benzofuroxane derivative (5 mmol), 2,4-diketone derivative $(10 \mathrm{mmol})$ and $500 \mathrm{mg}$ of solid support at room temperature to a $50 \mathrm{~mL}$ round-bottomed flask. After the reagents had dissolved, the solvent was then evaporated under reduced pressure and the mixture exposed to a CEM microwave (temperature $=60{ }^{\circ} \mathrm{C}$, power $=300 \mathrm{~W}$ ) for 3 periods of 5 min or more. After completion of the reaction, we added dichloromethane, and the solid support was removed by filtration. The crude compound was purified by column chromatography using dichloromethane:methanol (95:5).

All products were characterized by infrared (IR) and nuclear magnetic resonance (NMR) spectra. NMR was recorded on a Bruker Ultrashield $400 \mathrm{MHz}$ device, using Tetramethylsilane (TMS) as the internal standard and Dimethylsulfoside- $\mathrm{d}_{6}\left(\right.$ DMSO- $\left.\mathrm{d}_{6}\right)$ as a solvent. The chemical shifts are reported in ppm $(\delta)$, and coupling constant $(\mathrm{J})$ values are given in hertz $(\mathrm{Hz})$. Signal multiplicities are represented by s (singlet), d (doublet), t (triplet), c (quadruplet) and 
m (multiplet). The infrared (IR) spectra were performed on a Perkin Elmer 1600 FTIR in KBr Pellets. The frequencies are expressed in $\mathrm{cm}^{-}{ }^{1}$. Alugram1 SIL G/UV254 (Layer: $0.2 \mathrm{~mm}$ ) was used for thin layer chromatography.

Entry 1: Methyl 2-benzoyl-3-methylquinoxaline-7-carboxylate 1,4-di$N$-oxide. IR (KBr) v/cm ${ }^{-1}: 1726$ and $1674(\mathrm{C}=\mathrm{O}), 1328$ (N-oxide); ${ }^{1} \mathrm{H}-\mathrm{NMR}$ (DMSO-d $_{6}$ )/ppm: 2.32 (s, 3H, $\mathrm{CH}_{3}$ ), 3.99 (s, 3H, $\mathrm{CH}_{3} \mathrm{O}$ ), $7.59\left(\mathrm{t}, \mathrm{J}=7.67 \mathrm{~Hz}, 2 \mathrm{H}, \mathrm{H}_{3}\right.$ and $\left.\mathrm{H}_{5}, \mathrm{C}_{6} \mathrm{H}_{5}\right), 7.78(\mathrm{t}, \mathrm{J}=7.33 \mathrm{~Hz}, 1 \mathrm{H}$, $\left.\mathrm{H} 4, \mathrm{C}_{6} \mathrm{H}_{5}\right), 8.08\left(\mathrm{~d}, \mathrm{~J}=7.51 \mathrm{~Hz}, 2 \mathrm{H}, \mathrm{H}_{2}\right.$ and $\left.\mathrm{H}_{6}, \mathrm{C}_{6} \mathrm{H}_{5}\right), 8.37(\mathrm{~d}, \mathrm{~J}=$ $1.17 \mathrm{~Hz}, 1 \mathrm{H}, \mathrm{H}_{6}$ ), $8.49\left(\mathrm{~d}, 1 \mathrm{H}, \mathrm{H}_{5}\right), 8.96\left(\mathrm{~m}, 1 \mathrm{H}, \mathrm{H}_{8}\right)$.

Entry 2: Methyl 2-phenylcarbamoyl-3-methylquinoxaline-7-carboxylate 1,4-di-N-oxide. IR (KBr) v/cm ${ }^{-1}: 1728$ and $1684(\mathrm{C}=\mathrm{O}), 1336$

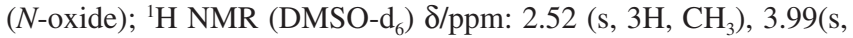
$3 \mathrm{H}, \mathrm{CH}_{3} \mathrm{O}$ ), 7.2 (t, J=7.38 Hz, $1 \mathrm{H}, \mathrm{NHC}_{6} \mathrm{H}_{5}$ ), 7.42 (t, J=7.78 Hz, $2 \mathrm{H}$, $\mathrm{H}_{3}$ and $\left.\mathrm{H}_{5}, \mathrm{NHC}_{6} \mathrm{H}_{5}\right), 7.67\left(\mathrm{~d}, \mathrm{~J}=7.92 \mathrm{~Hz}, 2 \mathrm{H}, \mathrm{H}_{2}\right.$ and $\left.\mathrm{H}_{6}, \mathrm{NHC}_{6} \mathrm{H}_{5}\right)$, $8.41\left(\mathrm{~d}, \mathrm{~J}=8.84 \mathrm{~Hz}, 1 \mathrm{H}, \mathrm{H}_{6}\right), 8.62\left(\mathrm{~d}, \mathrm{~J}=8.93 \mathrm{~Hz}, 1 \mathrm{H}, \mathrm{H}_{5}\right), 9.0(\mathrm{~s}$, $\left.1 \mathrm{H}, \mathrm{H}_{8}\right), 11.01$ (s, 1H, NH).

Entry 3: Dimethyl 3-methylquinoxaline-2,7-di-carboxylate 1,4-di$N$-oxide. IR ( $\mathrm{KBr})$ v/cm ${ }^{-1}$ : 2957 (ArC-H), $1716(\mathrm{C}=\mathrm{O}), 1320(\mathrm{~N}$ -

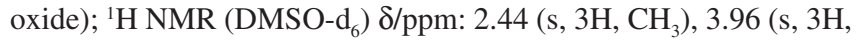
$\left.\mathrm{CH}_{3} \mathrm{OCO}\right), 4.04\left(\mathrm{~s}, 3 \mathrm{H}, \mathrm{COOCH}_{3}\right), 8.39$ (d, J=8.9 Hz, 1H, H $\mathrm{H}_{6}, 8.56$ (d, J=8.9 Hz, 1H, $\left.\mathrm{H}_{5}\right), 8.85\left(\mathrm{~s}, 1 \mathrm{H}, \mathrm{H}_{8}\right)$.

Entry 4: Ethyl methyl-3-methylquinoxaline-2,7-di-carboxylate 1,4-di$N$-oxide. IR (KBr) v/cm ${ }^{-1}: 1740$ and $1719(\mathrm{C}=\mathrm{O}), 1326$ ( $N$-oxide); ${ }^{1} \mathrm{H}$ NMR (DMSO-d $)$ ) $/ \mathrm{ppm}: 1.32\left(\mathrm{t}, 3 \mathrm{H}, \mathrm{CH}_{3} \mathrm{CH}_{2} \mathrm{O}\right) 2.45$ (s, 3H, $\mathrm{CH}_{3}$ ), 3.97 (s, 3H, $\mathrm{CH}_{3} \mathrm{O}$ ), 4.52 (c, $\left.2 \mathrm{H}, \mathrm{CH}_{3} \mathrm{CH}_{2} \mathrm{O}\right), 8.39$ (d, J= 1.80 and $\left.8.97 \mathrm{~Hz}, 1 \mathrm{H}, \mathrm{H}_{6}\right), 8.56\left(\mathrm{~d}, \mathrm{~J}=1.72 \mathrm{~Hz}, 1 \mathrm{H}, \mathrm{H}_{5}\right), 8.86\left(\mathrm{~m}, 1 \mathrm{H}, \mathrm{H}_{8}\right)$.

Entry 5: Tertbutyl methyl-3-methylquinoxaline-2,7-di-carboxylate 1,4-di-N-oxide. IR (KBr) v/cm ${ }^{-1}: 2976$ (ArC-H), 1732 and 1718 $(\mathrm{C}=\mathrm{O}), 1330$ ( $N$-oxide) ${ }^{1} \mathrm{H}$ NMR (DMSO-d ${ }_{6}$ ) $\delta / \mathrm{ppm}: 1.32$ (s, 9H, $\left(\mathrm{O}\left(\mathrm{CH}_{3}\right)_{3}\right), 2.45\left(\mathrm{~s}, 3 \mathrm{H}, \mathrm{CH}_{3}\right), 3.96\left(\mathrm{~s}, 3 \mathrm{H}, \mathrm{CH}_{3} \mathrm{O}\right), 8.36(\mathrm{t}, \mathrm{J}=9.4 \mathrm{~Hz}$, $\left.1 \mathrm{H}, \mathrm{H}_{6}\right), 8.58\left(\mathrm{~m}, 1 \mathrm{H}, \mathrm{H}_{5}\right), 8.88\left(\mathrm{~m}, \mathrm{~J}=13.2 \mathrm{~Hz}, 1 \mathrm{H}, \mathrm{H}_{8}\right)$.

Entry 6: Methyl 2-benzoyl-3-methylquinoxaline-7-carboxylate 1,4-di$N$-oxide. IR (KBr) v/cm ${ }^{-1}: 2949$ (ArC-H), 1726 and $1674(\mathrm{C}=\mathrm{O}), 1328$

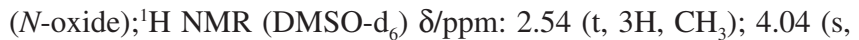
$\left.3 \mathrm{H}, \mathrm{CH}_{3} \mathrm{O}\right), 7.57\left(\mathrm{t}, J=8.1 \mathrm{~Hz}, 2 \mathrm{H}, \mathrm{H}_{3}\right.$ and $\left.\mathrm{H}_{5}, \mathrm{C}_{6} \mathrm{H}_{5}\right), 7.72(\mathrm{~s}, 1 \mathrm{H}$, $\left.\mathrm{H}_{4}, \mathrm{C}_{6} \mathrm{H}_{5}\right), 7.91\left(\mathrm{~d}, J=1.4 \mathrm{~Hz}, 2 \mathrm{H}, \mathrm{H}_{2} \mathrm{y} \mathrm{H}_{6}, \mathrm{C}_{6} \mathrm{H}_{5}\right), 8.52(\mathrm{~d}, J=9.0 \mathrm{~Hz}$, $\left.1 \mathrm{H}, \mathrm{H}_{6}\right), 8.76\left(\mathrm{~s}, 1 \mathrm{H}, \mathrm{H}_{5}\right), 9.23\left(\mathrm{~s}, 1 \mathrm{H}, \mathrm{H}_{8}\right)$.

Entry 7: Methyl 2-(2-thienylcarbonyl)-3-trifluoromethylquinoxaline7-carboxylate 1,4-di-N-oxide. IR (KBr) v/ $\mathrm{cm}^{-1}: 1732$ and $1664(\mathrm{C}=\mathrm{O})$,

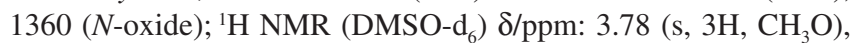
$7.32\left(\mathrm{~s}, 1 \mathrm{H}, \mathrm{C}_{4} \mathrm{H}_{3} \mathrm{~S}\right), 8.28\left(\mathrm{~d}, \mathrm{~J}=2.57 \mathrm{~Hz}, 2 \mathrm{H}, \mathrm{C}_{4} \mathrm{H}_{3} \mathrm{~S}\right), 8.5\left(\mathrm{~d}, 2 \mathrm{H}, \mathrm{H}_{5}\right.$ and $\left.\mathrm{H}_{6}\right), 8.96\left(\mathrm{~s}, 1 \mathrm{H}, \mathrm{H}_{8}\right)$.

Entry 8: Ethyl 2-benzoyl-3-methylquinoxaline-7-carboxylate 1,4-di$N$-oxide. IR (KBr) v/cm ${ }^{-1}: 1720$ and $1684(\mathrm{C}=\mathrm{O}), 1331$ ( $N$-oxide); ${ }^{1} \mathrm{H}$ NMR (DMSO-d $)_{6}$ ) $/$ ppm: $1.4\left(\mathrm{t}, \mathrm{J}=7.11 \mathrm{~Hz}, 3 \mathrm{H}, \mathrm{CH}_{3} \mathrm{CH}_{2} \mathrm{O}\right.$ ), 2.32 (s, 3H, $\left.\mathrm{CH}_{3}\right), 4.5$ (c, J=7.10 Hz, 2H, $\mathrm{CH}_{3} \mathrm{CH}_{2} \mathrm{O}$ ), 7.6 (t, J=7.79 Hz, $2 \mathrm{H}, \mathrm{H}_{3}$ and $\left.\mathrm{H}_{5}, \mathrm{C}_{6} \mathrm{H}_{5}\right), 7.8\left(\mathrm{t}, \mathrm{J}=7.43 \mathrm{~Hz}, 1 \mathrm{H}, \mathrm{H}_{4}, \mathrm{C}_{6} \mathrm{H}_{5}\right), 8.1(\mathrm{~d}, \mathrm{~J}=$ $7.35 \mathrm{~Hz}, 2 \mathrm{H}, \mathrm{H}_{2}$ and $\left.\mathrm{H}_{6}, \mathrm{C}_{6} \mathrm{H}_{5}\right), 8.4\left(\mathrm{~d}, \mathrm{~J}=1.72 \mathrm{~Hz}, 1 \mathrm{H}, \mathrm{H}_{6}\right), 8.5$ (s, $\left.1 \mathrm{H}, \mathrm{H}_{5}\right), 8.92\left(\mathrm{~m}, 1 \mathrm{H}, \mathrm{H}_{8}\right)$.

Entry 9: Ethyl 2-phenylcarbamoyl-3-methylquinoxaline-7-carboxylate 1,4-di-N-oxide. IR (KBr) v/cm ${ }^{-1}: 2982(\mathrm{ArC}-\mathrm{H}), 1726$ and 1684 $(\mathrm{C}=\mathrm{O}), 1333$ (N-oxide); ${ }^{1} \mathrm{H}$ NMR: 1.36-1.40 (m, 6H, $\mathrm{CH}_{3} \mathrm{CH}_{2} \mathrm{O}$ and $\left.\mathrm{CH}_{3}\right)$; 4.40-4.46 (m, 2H, $\left.\mathrm{CH}_{3} \mathrm{CH}_{2} \mathrm{O}\right), 7.2\left(\mathrm{t}, J=7.34 \mathrm{~Hz}, 1 \mathrm{H}, \mathrm{NHC}_{6} \mathrm{H}_{5}\right.$ ),
7.39-7.44 (m, $2 \mathrm{H}, \mathrm{H}_{3}$ and $\left.\mathrm{H}_{5}, \mathrm{NHC}_{6} \mathrm{H}_{5}\right)$, 7.64-7.66 (m, $2 \mathrm{H}, \mathrm{H}_{2}$ and $\left.\mathrm{H}_{6}, \mathrm{NHC}_{6} \mathrm{H}_{5}\right), 8.38-8.42\left(\mathrm{~m}, 1 \mathrm{H}, \mathrm{H}_{6}\right), 8.58-8.61\left(\mathrm{~m}, 1 \mathrm{H}, \mathrm{H}_{5}\right), 8.96$ (s, $\left.1 \mathrm{H}, \mathrm{H}_{8}\right), 11.10$ (s, 1H, NH).

Entry 10: Methyl ethyl -3-methylquinoxaline-2,7-di-carboxylate 1,4-di-N-oxide. IR (KBr) v/cm' 2982 (ArC-H), $1715(\mathrm{C}=\mathrm{O}), 1333$ (N-oxide); ${ }^{1} \mathrm{H}$ NMR (DMSO-d ${ }_{6}$ ) $\delta /$ ppm: 1.38 (s, 3H, $\left.\mathrm{CH}_{3} \mathrm{CH}_{2} \mathrm{O}\right), 2.64$ (s, $3 \mathrm{H}, \mathrm{CH}_{3}$ ), 3.99 (s, $3 \mathrm{H}, \mathrm{COOCH}_{3}$ ), 4.41 (s, $\left.2 \mathrm{H}, \mathrm{CH}_{3} \mathrm{CH}_{2} \mathrm{O}\right), 8.27$ $\left(\mathrm{m}, 1 \mathrm{H}, \mathrm{H}_{6}\right), 8.54\left(\mathrm{~s}, 1 \mathrm{H}, \mathrm{H}_{5}\right), 8.91\left(\mathrm{~s}, 1 \mathrm{H}, \mathrm{H}_{8}\right)$.

Entry 11: Ethyl 2-benzoyl-3-trifluoromethylquinoxaline-7-carboxylate 1,4-di-N-oxide. IR (KBr) v/cm $\mathrm{cm}^{-1}: 2987$ (ArC-H), 1735 (C=O), 1357 (N-oxide), 1261 and $1158\left(\mathrm{Ar}_{-} \mathrm{CF}_{3}\right) ;{ }^{1} \mathrm{H}$ NMR (DMSO-d $\left.{ }_{6}\right) \delta / \mathrm{ppm}: 1.4$ (t, $\left.J=7.11 \mathrm{~Hz}, 3 \mathrm{H}, \mathrm{CH}_{3} \mathrm{CH}_{2} \mathrm{O}\right) ; 4.5$ (c, $J=7.08 \mathrm{~Hz}, 2 \mathrm{H}, \mathrm{CH}_{3} \mathrm{CH}_{2} \mathrm{O}$ ), $7.6\left(\mathrm{~m}, 2 \mathrm{H}, \mathrm{H}_{3}\right.$ and $\left.\mathrm{H}_{5}, \mathrm{C}_{6} \mathrm{H}_{5}\right), 7.8\left(\mathrm{~m}, 1 \mathrm{H}, \mathrm{H}_{4}, \mathrm{C}_{6} \mathrm{H}_{5}\right), 8.2\left(\mathrm{~m}, 2 \mathrm{H}, \mathrm{H}_{2}\right.$ and $\left.\mathrm{H}_{6}, \mathrm{C}_{6} \mathrm{H}_{5}\right), 8.5\left(\mathrm{~m}, 2 \mathrm{H}, \mathrm{H}_{5}\right.$ and $\left.\mathrm{H}_{6}\right), 9.0\left(\mathrm{~m}, 1 \mathrm{H}, \mathrm{H}_{8}\right)$.

Entry 12: Ethyl 2-(2-thienylcarbonyl)-3-trifluoromethylquinoxaline7-carboxylate 1,4-di-N-oxide. IR ( $\mathrm{KBr}) \mathrm{v} / \mathrm{cm}^{-1}: 1726$ and $1665(\mathrm{C}=\mathrm{O})$,

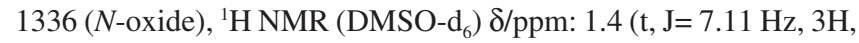
$\left.\mathrm{CH}_{3} \mathrm{CH}_{2} \mathrm{O}\right)$; $4.5\left(\mathrm{c}, \mathrm{J}=7.07 \mathrm{~Hz}, 2 \mathrm{H}, \mathrm{CH}_{3} \mathrm{CH}_{2} \mathrm{O}\right), 7.30-7.33\left(\mathrm{~m}, 1 \mathrm{H}, \mathrm{H}_{4}\right.$, $\mathrm{C}_{4} \mathrm{H}_{3} \mathrm{~S}$ ), 8.2 (dd, $\mathrm{J}=3.83$ and $0.76 \mathrm{~Hz}, \mathrm{H}_{5}, \mathrm{C}_{4} \mathrm{H}_{3} \mathrm{~S}$ ), 8.3 (dd, $\mathrm{J}=4.83$ and $\left.0.74 \mathrm{~Hz}, \mathrm{H}_{3}, \mathrm{C}_{4} \mathrm{H}_{3} \mathrm{~S}\right), 8.50-8.57\left(\mathrm{~m}, 2 \mathrm{H}, \mathrm{H}_{5}\right.$ and $\left.\mathrm{H}_{6}\right), 8.96\left(\mathrm{~m}, 1 \mathrm{H}, \mathrm{H}_{8}\right)$.

\section{ACKNOWLEDGMENTS}

We wish to express our gratitude to the "Fondo Mixto de Fomento a la Investigacion Cientifica y Tecnologica CONACyT - Gobierno del Estado de Tamaulipas, 2009-23" and the Universidad Autonoma de Tamaulipas and the Universidad de Navarra for their support in this study.

\section{REFERENCES}

1. Guillon, J.; Forfar, I.; Mamani-Matsudam, M.; Desplat, V.; Saliège, M.; Thiolat, D.; Massip, S.; Tabourier, A.; Léger, J. M.; Dufaure, B.; Haumont, G.; Jarry, C.; Mossalayi, D.; Bioorg. Med. Chem. 2007, 15, 194.

2. Tandon, V. K.; Yadav, D. B.; Maurya, H. K.; Chaturvedi, A. K.; Shukla, P. K.; Bioorg. Med. Chem. 2006, 14, 6120.

3. Romeiro, N. C.; Aguirre, G.; Hernández, P.; González, M.; Cerecetto, H.; Aldana, I.; Pérez-Silanes, S.; Monge, A.; Barreiro, E. J.; Lima, L. M.; Bioorg. Med. Chem. 2009, 17, 641.

4. Ortega, M. A.; Morancho, M. J.; Martínez-Crespo, F. J.; Sainz, Y.; Montoya, M. E.; López de Ceráin, A.; Monge, A.; Eur. J. Med. Chem. 2000, 35, 2.

5. Carta, A.; Corona, P.; Loriga, M.; Curr. Med. Chem. 2005, 12, 2259.

6. Amin, K. M.; Ismail, M. M. F.; Noaman, E.; Soliman, D. H.; Ammar, Y. A.; Bioorg. Med. Chem. 2006, 14, 6917.

7. Moreno, E.; Ancizu, S.; Perez-Silanes, S.; Torres, E.; Aldana, I.; Monge, A.; Eur. J. Med. Chem. 2010, 45, 4418.

8. Burguete A.; Pontiki, E.; Hadjipavlou-Litini, D.; Villar, R.; Vicente, E.; Solano, B.; Ancizu, S.; Perez-Silanes, S.; Aldana, I.; Monge, A.; Bioorg. Med. Chem. 2007, 17, 6439.

9. Monge, A.; Palop, J.; Senador, V.; Martínez-Crespo, F.; Sainz, Y.; Hamilton, E.; Barker, A.; Clarke, E.; Greenhow. D.; J. Med. Chem. 1995, $38,1786$.

10. Issidorides, C. H.; Haddadin, M. J.; J. Org. Chem. 1966, 31, 4067.

11. Zarranz, B.; Jaso, A.; Aldana, I.; Monge, A.; Bioorg. Med. Chem. 2004, 12,3711 .

12. Solano, B.; Junnotula, V.; Marin, A.; Villar, R.; Burguete, A.; Vicente, E.; Perez-Silanes, S.; Aldana, I.; Monge, A.; Dutta, S.; Sarkar, U.; Gates, K.; J. Med. Chem. 2007, 50, 5485. 
13. Talamás, F. X.; Smith, D. B.; Cervantes, A.; Franco, F.; Cutler, S. T.; Loughhead, D. G.; Morgans Jr, D. J.; Weikert, R. J.; Tetrahedron Lett. 1997, 38, 4725.

14. Lin, L.; Juanjuan, M.; Liuyan, J.; Yunyang, W.; J. Mol. Catal A: Chem. 2008, 291, 1 .

15. Kantam, M. L.; Jaya, V. S.; Lakshmi, M. J.; Reddy, B. R.; Choudary, B. M.; Bhargava, S. K.; Catal. Commun. 2007, 8, 1963.

16. Chankeshwara, S. V.; Chakraborti, A. K.; J. Mol. Catal A: Chem. 2006, $253,198$.

17. Habibi, D.; Marvi, O.; Catal. Commun. 2007, 8, 127.

18. Shimizu, K. I.; Higuchi, T.; Takasugi, E.; Hatamachi, T.; Kodama, T.; Satsuma, A.; J. Mol. Catal A: Chem. 2008, 284, 89.

19. Staszewska, A.; Stefanowicz, P.; Szewczuk, Z.; Tetrahedron Lett. 2005, $46,5525$.

20. Huang, T. K.; Wang, R.; Shi, L.; Lu, X. X.; Catal. Commun. 2008, 9 , 1143.
21. Ajaikumar, S.; Pandurangan, A.; Appl. Catal., A 2009, 357, 184.

22. Zhou, J. F.; Gong, G. X.; Shi, K.B.; Zhi, S.; J. Chinese Chemical Lett. 2009, 20, 672 .

23. Azizian, J.; Karimi, A. R.; Kazemizadeh, Z.; Mohammadi, A. A.; Mohammadizadeh, M. R.; Tetrahedron Lett. 2005, 46, 6155.

24. Zhao, Z.; Wisnoski, D. D.; Wolkenberg, S. E.; Leister, W. H.; Wang, Y.; Lindsley, C. W.; Tetrahedron Lett. 2004, 45, 4873.

25. Tohru, T.; Hiroaki, S.; Yusuke, S.; AC07D24136FI.

26. Hasegawa, M.; Takabatake, T.; Miyazama, T.; Yakushi 2001, 121, 379.

27. Nobuo, S.; Hiroaki, S.; Shinichi, M.; Tohru, T.; Heterocycles 2005, 65, 1589.

28. Hasegawa, M.; Takabatake, T.; Miyazawa, T.; ChemInform. 2001, 32, 379.

29. Yusuke, S.; Hiroaki, S.; Shinichi, M.; Tohru, T.; Heterocycles 2009, 78, 905. 